\title{
Pathological links between stroke and cardiac arrest
}

\author{
Shaila Ghanekar, Sydney Corey, Trenton Lippert and Cesar V. Borlongan*
}

\begin{abstract}
There may be a pathological connection between cardiac failure and ischemic stroke. In this article we describe pertinent research that demonstrates subsequent death of cardiac and neural myocytes in the post ischemic stroke brain. Current stroke therapy overlooks the connection between cardiac and cerebrovascular events and fails to address the shared risk factors. Current pre-clinical stroke investigations have provided evidence that suggests the presence of an indirect cell death pathway in which toxic molecules emanate from the stroke brain and trigger cardiac cell death. On the other hand, other studies highlight the presence of a reverse cell death cascade in which toxic molecules from the heart, following cardiac arrest, travel to the brain and induce ischemic cell death. Further examination of these putative cell death pathways between ischemic stroke and cardiac arrest will prompt the advancement of innovative treatments specifically targeting both diseases, leading to ameliorated clinical results of patients diagnosed with heart failure and ischemic stroke.
\end{abstract}

Keywords: Cerebral ischemia, Heart disease, Stem cells

\section{Background}

Stroke and cardiovascular disease are two of the principle causes of death in the world. In total, the United States allots $\$ 260.7$ billion to address both cerebrovascular and cardiac disease every year $[1,2]$. The two diseases share several predisposing factors including obesity, diabetes, hypertension, high cholesterol, and family history of heart disease [3-6]. It is worth noting that $2-6 \%$ of all deaths originating from a cardiac condition took place roughly 3 months following a cerebral infarction [7, 8]. This calculation is reinforced by evidence that demonstrates a higher likelihood of cardiac death following ischemic stroke. Data show that following an ischemic stroke, the patient's body has increased volumes of plasma catecholamines and cardiac enzymes $[7,8]$. This increase in cardiac enzymes is coincident with increased levels of troponin and creatine phosphokinase, which are a signal of stress or death in cardiac cells $[7,8]$. In addition, the elevated level of catecholamine is linked to high blood pressure under stressful conditions. [9-11]. Furthermore, inflammation following ischemic stroke is also indicative of a pathological pathway

\footnotetext{
* Correspondence: cborlong@health.usf.edu Center of Excellence for Aging and Brain Repair, Department of Neurosurgery and Brain Repair, University of South Florida Morsani College of Medicine, 12901 Bruce B. Downs Blvd, Tampa 33612, FL, USA
}

connecting the brain and the heart. C-reactive protein (CRP), which is elevated during periods of inflammation within the body, was the specific factor found to be associated with the risk of new cardiovascular events in stroke patients [12], in thatpatients with higher levels of CRP following ischemic stroke isplayed $36 \%$ greater risk of a new cardiovascular event [12]. This implicates inflammation as a key factor in the cascading effect of harmful substances from the stroke brain to the cardiovascular system.

Although the association between cardiac and cerebrovascular events has been made apparent, clinics continue to overlook the correlations between the two. Regrettably, up to $88 \%$ of all patients who suffer from an ischemic stroke display cardiac symptoms within 4 weeks after the stroke [13]. Ischemic damage in the insular cortex yields the highest occurrence of cardiac death in comparison to other regions of the brain [13]. While it is widely accepted that the insular cortex preludes cardiac myocyte death, it has yet to be determined whether myocyte death is caused by a sub-region or the entire insular cortex. As a result, the connection between the insular cortex and heart disease has yet to be firmly established.

Aware of the correlation between cardiac failure and ischemic stroke, we decided to observe the connection between neural cell death after ischemic stroke and cardiac 
myocyte compromise, utilizing both in vitro and in vivo stroke models [14]. In the in vitro study, we applied the method of oxygen-glucose deprivation (OGD) to primary rat neuronal cells (PRNCs), determining cellular alterations in rat cardiac myocytes (RCMs) after the ischemicreperfusion injury using the supernatant. For the in vivo study, transient middle cerebral artery occlusion (MCAo) was used in adult rats. Immunohistochemical analyses were performed on both the hearts and brains of the rats to determine the various cell death markers. We predicted that the release of cell death factors by the ischemic brain would result in cardiac myocyte compromise. These results demonstrated that cerebral infarction cascaded cell death signals upon the heart (Fig. 1) [14]. Our results from the in vitro study revealed that the PRNCs caused toxic effects in the RCMs. The in vivo study supported these findings, revealing that animals subject to ischemic stroke experienced RCM cell death. These findings suggest a close pathological link between cerebral ischemia and cardiac arrest.

Several conditions, including cerebral ischemia and cardiac failure, have been linked to other major neurological

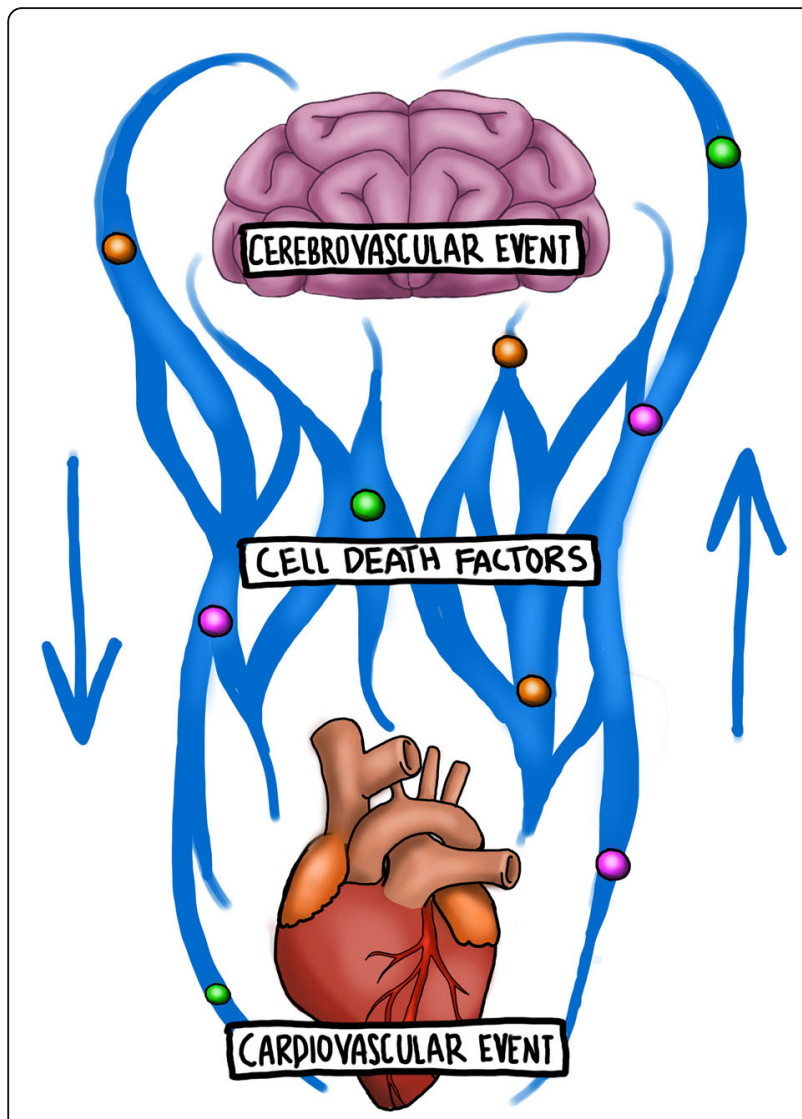

Fig. 1 Cell death factor cascade represents the pathological link between the brain and the heart. Ischemic stroke can lead to detrimental effects on the heart. Cardiac arrest can also release cell death factors to the brain potentially leading to ischemic stroke disabilities across the globe. Situations where both cerebral and cardiac conditions develop concurrently result in high mortality rates [15]. Our recent investigation demonstrated cardiac myocyte death following cerebral ischemia, further supporting the assertion that ischemic stroke can possibly result in cardiac complications [14]. Physical symptoms can be indicative of a detrimental effect on cardiac function immediately following the cerebrovascular event, which may predict the occurrence of a myocardial infarction [16]. Indeed, $5.7 \%$ of patients presented with arterial fibrillation or flutter within 5 days of stroke [16] suggesting that the stroke brain and the corresponding heart dysfunctions may be pathologically linked.

These findings prompt the question "How does ischemic stroke interrupt normal cardiac function?" Although the brain is responsible for controlling the heart rate, the mechanism of beating occurs naturally. When the brain is subjected to ischemic stroke, its ability to control heart rhythm is disrupted, directly compromising the heart $[8,13]$. As previously noted, the insular cortex of the brain is the region primarily responsible for controlling the heart rate [17]. Collectively, these observations suggest a crosstalk between the heart and insular cortex shortly after cerebral ischemia.

Shockingly, our findings revealed that the induced direct ischemia/reperfusion ( $\mathrm{I} / \mathrm{R})$ damage to the RCMs in fact had no impact on cardiac myocyte death [14]. After adding the OGD supernatant, our results from the in vitro study demonstrated massive cardiac cell death, suggesting that an indirect pathway originating from the area of cerebral ischemia and involving brain-mediated cell death signals was responsible for the observed cardiac changes. While solid evidence has been found, it is necessary to consider other possible parallel cell death pathways that connect cardiac cell death and ischemic stroke, such as neuroendocrine mediated myocardial suppression [7, 18], systemic responses $[6,19,20]$, and alterations in circulating endothelial progenitor cells [21].

Further immunocytochemical evaluations demonstrated that OGD-exposed PRNCs displayed a substantial timedependent proliferation of positive cells against all cell death markers $24 \mathrm{~h}$ after $\mathrm{I} / \mathrm{R}$ damage in comparison to the control group [14]. Furthermore, cerebral ischemic events significantly modified the autophagic pathway rather than the necrotic and apoptotic cell death mechanisms, creating toxic effects to the heart [14], suggesting that autophagyrelated cell death signals could be primarily responsible for cardiac injury following stroke.

More in depth examination of our in vivo findings revealed that both the heart and brain also displayed all the death cell markers of necrosis, autophagy, and apoptosis [14]. In addition, not only were apoptotic and autophagic cells identified in the ipsilateral side, but also on the contralateral side. These findings indicate that 
while the initial injury was contained within one hemisphere, the damage can spread globally to the rest of the brain as the disease progresses, and can possibly reach the heart.

Oppenheimer and Hachinski advanced the idea of the lateralization of stroke resulting in cardiac complications [7] and it continues to be a very prevalent topic for researchers [22]. In normal humans, lateralization of cardiovascular autonomic function has been demonstrated in the forebrain, notably injury to the insular cortex correlates with more prominent autonomic imbalance, leading to arrhythmias and even sudden death [23]. In connection, the knowledge that left-handers have a lower possibility of sudden death in comparison to right handers [23] implies that an ischemic stroke to the right hemisphere is more responsible for arrhythmias than a stroke to the left hemisphere. While a comparable lateralization model has been demonstrated in insular epilepsy-dysphoric responses were statistically related to stimulation in the right hemisphere rather than stimulation in the mesial frontal, orbitofrontal, mesial temporal, and insular stimulation sites in the left hemisphere [24] - it has also been disputed in studies on ictal bradycardia, an occurrence associated with bilateral hemispheric seizure activity instead of a lateralized sign that localizes seizure onset. These findings challenge the notion that a primarily unilateral parasympathetic cardiomotor representation exists in the left hemisphere [25].

In extreme ischemic events, brain natriuretic peptide (BNP), which acts as a biomarker that correlates with high mortality rates, levels can be raised [26]. BNP is released by cardiac muscle under increased amounts of stress and is linked to a defect in the left ventricle as well as the severity of the defect. It also participates in natriuresis and vasodilation, decreasing systolic blood pressure [26]. In ischemic stroke, it has been shown that all-cause mortality is linked to BNP and N-terminal-probrain natriuretic peptide (NT-proBNP), as well as being closely associated with cardio-embolic stroke. This evidence suggests that the kidney, along with the heart, is connected with stroke pathology. Along with all-cause mortality in ischemic stroke, transient ischemic attack (TIA) or hemorrhagic stroke is associated with an elevation of NT-proBNP and BNP levels, conveying a relationship between the heart and ischemic stroke. Altogether, these investigations suggest that an elevation of BNP levels is related to a worse outcome after stroke [27]. In addition, these studies reveal that renal failure is connected to an increase of NT-proBNP and BNP, both functioning as independent predictors of death due to kidney failure and as risk factors for ischemic stroke together with cardiac failure.

The idea that an indirect pathological pathway of cell death exists, initiating in the brain and concluding in the heart, suggests that there must be soluble molecules that are produced by the ischemic brain cells that could carry cell death signals to cardiac myocytes. However, these toxic molecules have yet to be determined. In addition, as these toxins move through the cardiovascular system, it is necessary to conduct investigations regarding their effects on vital peripheral organs. At its most basic of assertions, our study [14] suggests that a cardiac exam should be considered following an ischemic stroke and could potentially be integrated into standard clinical stroke treatment and management.

That cerebral ischemia may result in cardiac myocyte death, complements a series of investigations that show the reverse interaction, whereby heart events lead to stroke-like brain pathology [15, 28-30]. A biological crosstalk involving the brain and the heart is corroborated by evidence demonstrating cardiac events result in a strokelike brain pathology. The exact biological mechanisms and specific pathways linking myocardial infarction and brain damage are not fully understood, but accumulation of intracellular calcium, mitochondrial overload, and impaired ATP production have been observed in neuronal cell death following cardiac arrest [31]. Most notably, inflammatory mechanisms similarly play a large part in post-cardiac arrest leading to cerebral injury. Following myocardial infarction, the immune-privileged microenvironment that exists in the brain is disrupted and characterized by activation of microglia, migration of nearby macrophages and neutrophils, as well as the release of pro-inflammatory cytokines [32].

\section{Conclusions}

The merging of these studies on cardiac arrest and ischemic stroke strongly implies the presence of a pathological pathway connecting these two diseases (Fig. 1). Further investigations and understanding of overlapping molecular, cellular, and anatomical alterations seen in heart failure and ischemic stroke will guide the development of innovative treatment strategies focused on combatting both diseases, which will likely lead to improved clinical outcomes of patients afflicted with both illnesses.

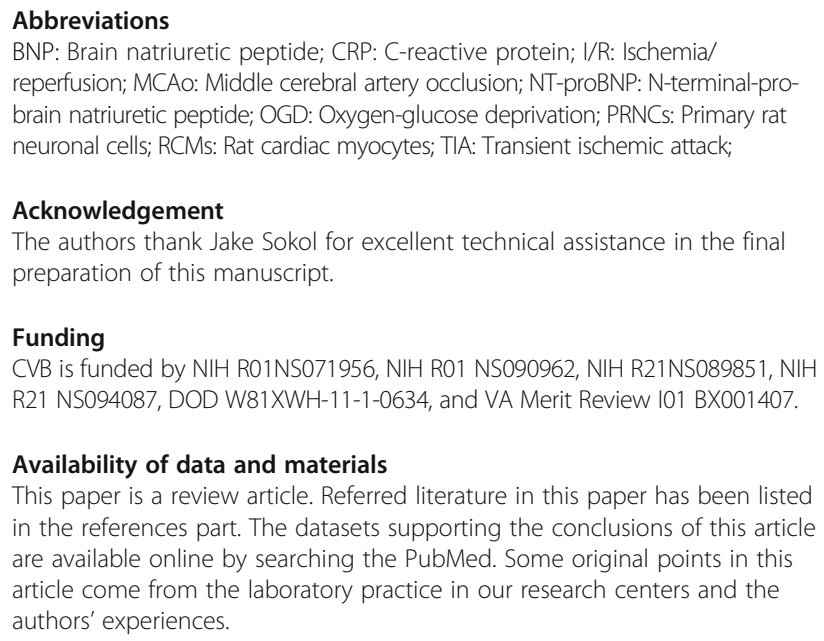

Acknowledgement

The authors thank Jake Sokol for excellent technical assistance in the final preparation of this manuscript

\section{Funding}

CVB is funded by NIH R01NS071956, NIH R01 NS090962, NIH R21NS089851, NIH R21 NS094087, DOD W81XWH-11-1-0634, and VA Merit Review 101 BX001407.

\section{Availability of data and materials}

This paper is a review article. Referred literature in this paper has been listed in the references part. The datasets supporting the conclusions of this article are available online by searching the PubMed. Some original points in this article come from the laboratory practice in our research centers and the authors' experiences. 


\section{Authors' contributions}

The authors contributed equally to the conceptualization and write-up of this manuscript.

\section{Competing interests}

The authors declare no competing interests.

\section{Consent for publication}

All authors approved the publication of this manuscript.

\section{Ethics approval and consent to participate}

Not applicable.

Received: 14 July 2016 Accepted: 12 September 2016

Published online: 01 December 2016

\section{References}

1. Centers for Disease Control and Prevention. Prevalence of coronary heart disease-United States, 2006-2010. MMWR Morb Mortal Wkly Rep. 2011;60:1377-81.

2. Centers for Disease Control and Prevention. Prevalence of stroke-UnitedStates, 2006-2010. MMWR Morb Mortal Wkly Rep. 2012;61:379-82

3. Kannel WB. The Framingham Study: ITS 50-year legacy and future promise. J Atheroscler Thromb. 2000;6:60-6.

4. Lo EH, Dalkara T, Moskowitz MA. Mechanisms, challenges and opportunities in stroke. Nat Rev Neurosci. 2003:4:399-415.

5. Zhang ZG, Chopp M. Neurorestorative therapies for stroke: underlying mechanisms and translation to the clinic. Lancet Neurol. 2009;8:491-500.

6. Lawlor DA, Smith GD, Leon DA, Sterne JA, Ebrahim S. Secular trends in mortality by stroke subtype in the 20th century: a retrospective analysis. Lancet. 2002;360:1818-23.

7. Oppenheimer SM, Hachinski VC. The cardiac consequences of stroke. Neurol Clin. 1992:10:167-76.

8. Prosser J, Macgregor L, Lees KR, Diener HC, Hacke W, Davis S. Predictors of early cardiac morbidity and mortality after ischemic stroke. Stroke. 2007;38: 2295-302.

9. Klingelhofer J, Sander D. Cardiovascular consequences of clinical stroke. Baillieres Clin Neurol. 1997;6:309-35.

10. Adams 3rd JE, Abendschein DR, Jaffe AS. Biochemical markers of myocardial injury. Is MB creatine kinase the choice for the 1990? Circulation. 1993;88:750-63.

11. Snider SR, Kuchel O. Dopamine: an important neurohormone of the sympathoadrenal system: significance of increased peripheral dopamine release for the human stress response and hypertension. Endocr Rev. 1983:4:291-309.

12. Napoli M, Papa F. Inflammation, hemostatic markers, and antithrombotic agents in relation to long-term risk of new cardiovascular events in first-ever ischemic stroke patients. Stroke. 2002;33:1763-71.

13. Ay H, Koroshetz WJ, Benner T, Vangel MG, Melinosky C, Arsava EM, et al. Neuroanatomic correlates of stroke-related myocardial injury. Neurology. 2006;66:1325-9

14. Ishikawa H, Tajiri N, Vasconcellos J, Kaneko Y, Mimura O, Dezawa M, et al. Ischemic stroke brain sends indirect cell death signals to the heart. Stroke. 2013;44:3175-82.

15. Go AS, Mozaffarian D, Roger VL, Benjamin EJ, Berry JD, Borden WB, et al. Heart disease and stroke statistics - 2013 update: a report from the American Heart Association. Circulation. 2013;127:e6-e245.

16. Jabaudon D, Sztajzel J, Sievert K, Landis T, Sztajzel R. Usefulness of ambulatory 7-day ECG monitoring for the detection of atrial fibrillation and flutter after acute stroke and transient ischemic attack. Stroke. 2004;35:1647-51.

17. Korpelainen JT, Sotaniemi KA, Myllyla W. Autonomic nervous system disorders in stroke. Clin Auton Res. 1999;9:325-33.

18. Wira 3rd CR, Rivers E, Silver B, Lewandowski C. The impact of cardiac contractility on cerebral blood flow in ischemia. West J Emerg Med. 2011;1:227-32.

19. Kostulas N, Kivisakk P, Huang Y, Matusevicius D, Kostulas V, Link H. Ischemic stroke is associated with a systemic increase of blood mononuclear cells expressing interleukin-8 mRNA. Stroke. 1998;29:462-6.

20. Mo X, Li T, Ji G, Lu W, Hu Z. Peripheral polymorphonuclear leukocyte activation as a systemic inflammatory response in ischemic stroke. Neurol Sci. 2013;34:1509-16.
21. Paczkowska E, Golab-Janowska M, Bajer-Czajkowska A, Machalinska A, Ustianowski P, Rybicka M, et al. Increased circulating endothelial progenitor cells in patients with haemorrhagic and ischaemic stroke: the role of endothelin-1. J Neurol Sci. 2013:325:90-9.

22. Inamasu J, Sugimoto $K$, Watanabe E, Kato $Y$, Hirose $Y$. Effect of insular injury on autonomic functions in patients with ruptured middle cerebral artery aneurysms. Stroke. 2013;44:3550-2.

23. Ozdemir $\mathrm{O}$, Hachinski V. Brain lateralization and sudden death: its role in the neurogenic heart syndrome. J Neurol Sci. 2008;268:6-11.

24. Smith JR, Lee GP, Fountas K, King DW, Jenkins PD. Intracranial stimulation study of lateralization of affect. Epilepsy Behav. 2006;8:534-41.

25. Britton JW, Ghearing GR, Benarroch EE, Cascino GD. The ictal bradycardia syndrome: localization and lateralization. Epilepsia. 2006;47:737-44.

26. Shibazaki K, Kimura K, Okada Y, Iguchi Y, Terasawa Y, Aoki J. Heart failure may be associated with the onset of ischemic stroke with atrial fibrillation: a brain natriuretic peptide study. J Neurol Sci. 2009;281:55-7.

27. Montaner J, Perea-Gainza M, Delgado P, Ribó M, Chacón P, Rosell A, et al. Etiologic diagnosis of ischemic stroke subtypes with plasma biomarkers. Stroke. 2008:39:2280-7.

28. Devries AC, Craft TK, Glasper ER, Neigh GN, Alexander JK. 2006 Curt P. Richter award winner: social influences on stress responses and health. Psychoneuroendocrinology. 2007;32:587-603.

29. Ning M, Lo EH, Ning PC, Xu SY, Mcmullin D, Demirjian Z, et al. The brain's heart-therapeutic opportunities for patent foramen ovale (PFO) and neurovascular disease. Pharmacol Ther. 2013;139:111-23.

30. Micheli S, Agnelli G, Caso V, Alberti A, Palmerini F, Venti M, et al. Acute myocardial infarction and heart failure in acute stroke patients: frequency and influence on clinical outcome. J Neurol. 2012;259:106-10.

31. Uchino H, Ogihara Y, Fukui $H$, et al. Brain injury following cardiac arrest: pathophysiology for neurocritical care. J Intensive Care. 2016;4:31.

32. Xiang $\mathrm{Y}$, Zhao $\mathrm{H}$, Wang J, et al. Inflammatory mechanisms involved in brain injury following cardiac arrest and cardiopulmonary resuscitation. Biomed Rep. 2016;5(1):11-7.

\section{Submit your next manuscript to BioMed Central and we will help you at every step:}

- We accept pre-submission inquiries

- Our selector tool helps you to find the most relevant journal

- We provide round the clock customer support

- Convenient online submission

- Thorough peer review

- Inclusion in PubMed and all major indexing services

- Maximum visibility for your research

Submit your manuscript at www.biomedcentral.com/submit
Biomed Central 